\title{
Hidden Paradoxes in Generic Drug Substitution Affecting Pharmacotherapy
}

\author{
A. Sapone, D. Canistro, B. Bonamassa, G. L. Biagi, and M. Paolini \\ Molecular Toxicology Unit, Department of Pharmacology, Alma Mater Studiorum, University of Bologna, \\ Via Irnerio 48, 40126 Bologna, Italy \\ Correspondence should be addressed to A. Sapone, andrea.sapone@unibo.it \\ Received 13 February 2009; Accepted 21 April 2009 \\ Copyright (c) 2009 A. Sapone et al. This is an open access article distributed under the Creative Commons Attribution License, \\ which permits unrestricted use, distribution, and reproduction in any medium, provided the original work is properly cited.
}

The issue of the generic versus brand-name drug interchangeability is an intensely debated theme historically focused on whether bioequivalence testing can guarantee therapeutic efficacy. The controversy is supported by reports of the lack of therapeutic equivalence between some generic medicinal products and brand-name drugs, as well as by a great deal of editorials expressing a negative view on generic drug substitution. This could be of particular concern for critical therapeutic categories such as psychotropic, cardiovascular, and metabolic/endocrine drugs as well as for special subpopulations such as elderly, debilitated/psychoneurotic patients, infants and children $[1,2]$. To deal with this issue, large, prospective controlled evaluations have been proposed, providing information on how current bioequivalence and pharmacological equivalence translate into clinical equivalence [3]. In our opinion, however, although on one hand this strategy could be suitable in clinical trials to ascertain the "essential similarity" requirement in drug substitution - in terms of active ingredient (amount and type), route of administration, and therapeutic effectiveness - on the other hand, it might be insufficient in real practice to support the therapeutic equivalence for the general population. This could also be due to the possible influence of the marketing to patient's response.

To better explain what we are saying, let us recall what follows. Our knowledge on the placebo effect, a phenomenon that has intrigued scientists since 1955 when Henry K. Beecher published the article "The powerful placebo" [4] and quantified the placebo effect [5], has evolved from being thought of as a nuisance in pharmacological intervention to the mechanistic aspects of its incontrovertible function. Two main models of how the placebo-or placebo related effects—works have been proposed. The "expectance theory," which is essentially based on the patient's expectation of clinical benefits occurring in association with the verbal suggestion that clinical improvement will soon occur; the "classic conditioning" model foresees that after repeated associations between a conditioned stimulus (e.g., colour and shape of a pill) and an unconditioned stimulus (e.g., the active agent in the pill), the first stimulus alone may induce a conditioned response similar to the one induced by the active drug [6].

There is no doubt that, in randomized, double-blind controlled studies, the contribution of the placebo effect to therapeutic efficacy is "equally distributed" between patients receiving the generic product and the ones taking the brand name with an overall nondiscriminating therapeutic role between these two patient classes. In this case, identical medical information and "apparently" identical medicaments are, indeed, given. However, in real practice, the frequent choice of the cheaper drug over the generic drug, ignoring physician recommendations for the brand name (contradicting the expectation theory) and/or the total or partial lack in the generic product of conventional perceptual characteristics affecting patient expectation (contradicting the classic conditioning theory), such as preparation form (e.g., capsule or pill), size, colour, taste, and packaging [7], all regularly found in the brand name, may totally or partially cancel the placebo effect.

This phenomenon is reinforced by commercial feature changes. The term "generic" evokes a poor product quality so that, if compared to the term "brand" evoking the gold standard (like designer clothes), it can subsequently reduce the placebo contribution to therapeutic efficacy. It is fairly 
significant that a recent trial on the placebo responses to commercial variables showed that the medicines believed to be less expensive (discounted) proved less effective than the regular price ones [8]. So, paradoxically, the "conscious" choice of the generic product because of economic benefits can "unconsciously" reduce the efficacy of the medical therapy.

Although approximately $50 \%$ of pharmacy prescriptions in the USA and EU are filled with generic drugs, resulting in considerable cost savings, indiscriminate switching among generic products should be avoided. This is especially true in the above-mentioned critical cases and/or in conditions associated with a strong placebo response [6]. In the meantime, an harmonization of the various perceptional characteristics between brand-name and generic products by pharmaceutical companies-a choice pointing to drug effectiveness rather than marketing-would maximize the treatment benefits.

Conflict of Interest Statement. The authors declare that there are no conflicts of interest.

\author{
A. Sapone \\ D. Canistro \\ B. Bonamassa \\ G. L. Biagi \\ M. Paolini
}

\title{
References
}

[1] P. Meredith, "Bioequivalence and other unresolved issues in generic drug substitution," Clinical Therapeutics, vol. 25, no. 11, pp. 2875-2890, 2003.

[2] A. S. Kesselheim, A. S. Misono, J. L. Lee, et al., "Clinical equivalence of generic and brand-name drugs used in cardiovascular disease: a systematic review and meta-analysis," Journal of the American Medical Association, vol. 300, no. 21, pp. 2514-2526, 2008.

[3] M. Van Ameringen, C. Mancini, B. Patterson, and M. Bennett, "Symptom relapse following switch from Celexa to generic citalopram: an anxiety disorders case series," Journal of Psychopharmacology, vol. 21, no. 5, pp. 472-476, 2007.

[4] H. K. Beecher, "The powerful placebo," Journal of the American Medical Association, vol. 159, no. 17, pp. 1602-1606, 1955.

[5] G. S. Kienle and H. Kiene, "The powerful placebo effect: fact or fiction?" Journal of Clinical Epidemiology, vol. 50, no. 12, pp. 1311-1318, 1997.

[6] F. Benedetti, "Mechanisms of placebo and placebo-related effects across diseases and treatments," Annual Review of Pharmacology and Toxicology, vol. 48, pp. 33-60, 2008.

[7] A. R. Rao and K. B. Monroe, "The effect of price, brand name, and store name on buyers' perceptions of product quality: an integrative review," Journal of Marketing Research, vol. 26, no. 3, pp. 351-357, 1989.

[8] R. L. Waber, B. Shiv, Z. Carmon, and D. Ariely, "Commercial features of placebo and therapeutic efficacy," Journal of the American Medical Association, vol. 299, no. 9, pp. 1016-1017, 2008. 

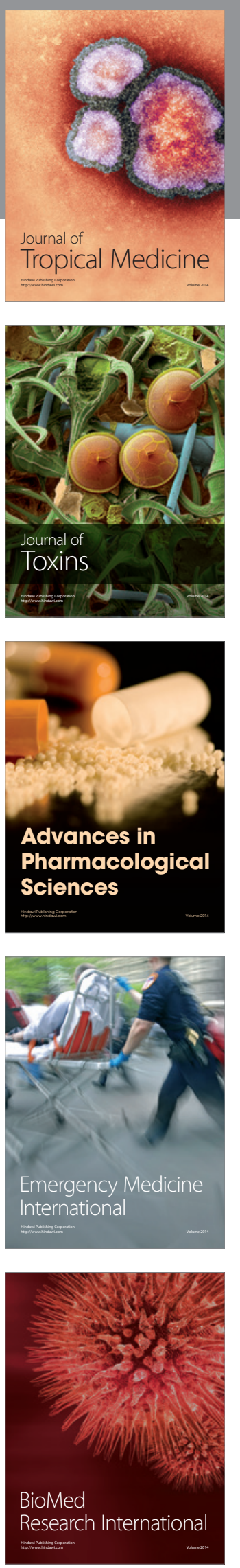
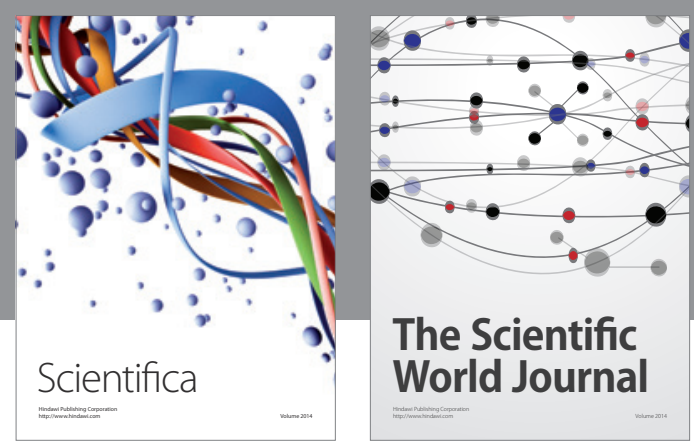

The Scientific World Journal
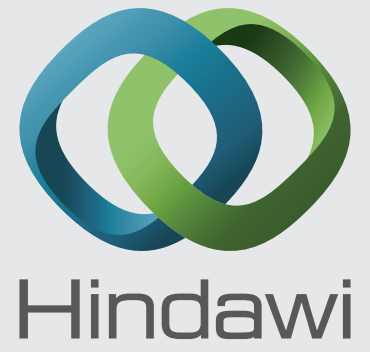

Submit your manuscripts at

http://www.hindawi.com
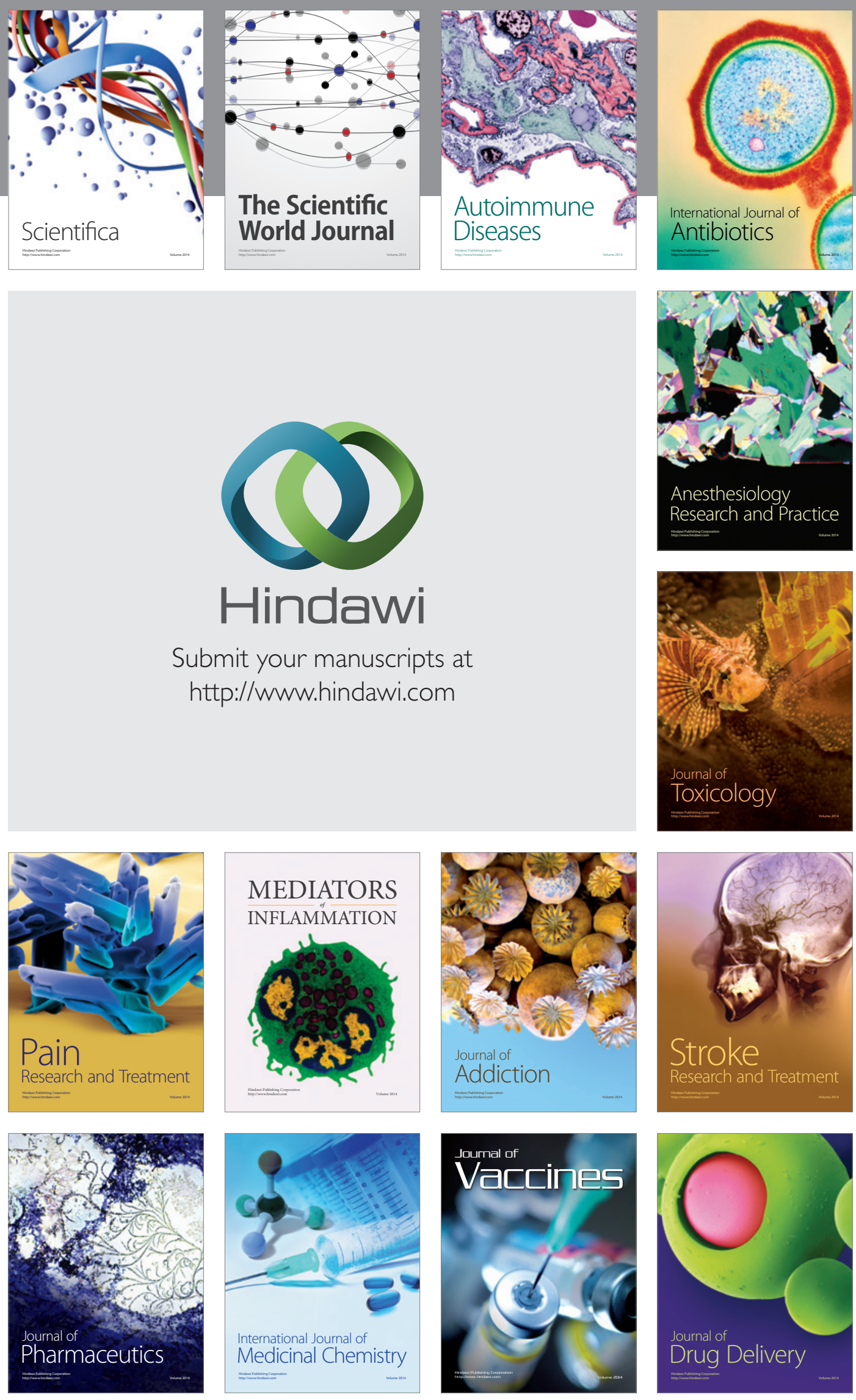